\title{
Fingerprint Verification Using Local Interest Points and Descriptors*
}

\author{
Javier Ruiz-del-Solar, Patricio Loncomilla, and Christ Devia \\ Department of Electrical Engineering, Universidad de Chile
}

\begin{abstract}
A new approach to automatic fingerprint verification based on a general-purpose wide baseline matching methodology is here proposed. The approach is not based on the standard ridge-minutiae-based framework. Instead of detecting and matching the standard structural features, local interest points are detected in the fingerprints, then local descriptors are computed in the neighborhood of these points, and afterwards these descriptors are compared using local and global matching procedures. Then, a final verification is carried out by a Bayes classifier. The methodology is validated using the FVC2004 dataset, where competitive results are obtained.
\end{abstract}

Keywords: Fingerprint verification, Wide Baseline Matching, SIFT.

\section{Introduction}

Fingerprint verification is one of the most employed biometric technologies. A fingerprint is the pattern of ridges and furrows on the surface of a fingertip. It is formed by the accumulation of dead, cornified cells [5]. The fingerprint pattern is unique and determined by the local ridge characteristics and the presence of ridge discontinuities, called minutiae. The two most prominent minutiae are ridge termination and ridge bifurcation. Minutiae in fingerprints are generally stable and robust to fingerprint impression conditions. Singular points, called loop and delta, are a sort of control points around which the ridge-lines are "wrapped" [10]. Many approaches to automatic fingerprint verification have been proposed in the literature and the research on this topic is still very active. In most of the cases the automatic verification process is based on the same procedure employed by human experts: (i) Detection of structural features (ridges, minutiae, and/or singular points), and in some cases derived features as the orientation field, which allow characterizing the fingerprints, and (ii) Comparison between the features in the input and reference fingerprints. This comparison is usually implemented using minutiae-based matching, ridge pattern comparison and/or correlation between the fingerprints. The mentioned comparison methodologies can be described as [10]: Minutiae-based matching: It consists of finding the alignment between the input and the reference minutiae sets that results in the maximum number of minutiae pairings; Ridge feature-based matching: The approaches belonging to this

* This research was funded by Millennium Nucleus Center for Web Research, Grant P04-067-F, Chile. 
family compare fingerprints in term of features extracted from the ridge pattern (e.g. local orientation and frequency, ridge shape, texture information); and Correlationbased matching: Two fingerprint images are superimposed and the correlation (at the intensity level) between corresponding pixels is computed for different alignments (e.g., various displacements and rotations).

In state of the art fingerprint verification systems several structural features and comparison methodologies are jointly employed. For instance, in the 2004 Fingerprint Verification Competition (FVC2004) the 29 participants (from 43) that provided algorithm's information employed the following methodologies [2]: (a) Features: minutiae (27), orientation field (19), singular points (12), ridges (10), local ridge frequency (8), ridge counts (6), raw or enhanced image parts (4), and texture measures (3); and (b) Comparison methodology: minutiae global (20), minutiae local (15), correlation (7), ridge pattern geometry (5), and ridge pattern texture (2).

In this general context the main objective of this article is to propose a new approach to automatic fingerprint verification that it is not based on the standard ridge-minutiae-based framework, but in a general-purpose wide baseline matching methodology. Instead of detecting and matching the standard structural features, in the proposed approach local interest points are detected in the fingerprint, then local descriptors are computed in the neighborhood of these points, and finally these descriptors are compared using local and global matching procedures. The local interest points do not correspond to minutiae or singular points, but to local maxima in a scale-space representation of the fingerprint image.

The main intention in proposing this new approach is to show an alternative procedure for solving the fingerprint verification procedure. We believe that this new approach can complement and enrich the standard procedures, and it can be used in addition to them. In this sense, we are not proposing a methodology for replacing the standard one, but a complementary solution. This article is structured as follows. In section 2 the employed methodology for solving the general wide-baseline matching problem is described. In section 3 is presented the adaptation of this methodology to fingerprint verification. In section 4 are presented results of this new approach for fingerprint verification. Finally, in section 5 some conclusions of this work are given.

\section{Wide Baseline Matching Using Local Interest Points}

Wide baseline matching (object recognition) approaches based on local interest points (invariant features) have become increasingly popular and have experienced an impressive development in the last years [3][7][8][11][15]. Typically, local interest points are extracted independently from both a test and a reference image, then characterized by invariant descriptors, and finally the descriptors are matched until a given transformation between the two images is obtained. Most employed local detectors are the Harris detector [4] and the Lowe's sDoG+Hessian detector [7], being the Lowe's detector multiscale and the Harris detector single scale. Best performing affine invariant detectors are the Harris-Affine and the Hessian-Affine [13], but they are too slow to be applied in general-purpose applications. The most popular and best performing invariant descriptor [12] is the SIFT (Scale Invariant Feature Transform) [7]. For selecting the local detector and invariant descriptor to be used in a given 
application it should be taken into account the algorithm's accuracy, robustness and processing speed. Lowe's system [7] using the SDoG+Hessian detector, SIFT descriptors and a probabilistic hypothesis rejection stage is a popular choice, given its recognition capabilities, and near real-time operation. However, Lowe's system main drawback is the large number of false positive detections. This is a serious problem when using it in real world applications.

One of the main weaknesses of Lowe's algorithm is the use of just a simple probabilistic hypothesis rejection stage, which cannot successful reduce the number of false positives. Loncomilla and Ruiz-del-Solar (L\&R) propose a system that reduces largely the number of false positives by using several hypothesis rejection stages [8][9]. This includes a fast probabilistic hypothesis rejection stage, a linear correlation verification stage, a geometrical distortion verification stage, a pixel correlation verification stage, a transformation fusion procedure, and the use of the RANSAC algorithm and a semi-local constraints test. In [9] are compared the Lowe's and the L\&R systems using 100 pairs of real-world high-textured images (variations in position, view angle, image covering, partial occlusion, in-plane and out-of the-plane rotation). The results show that in this dataset the L\&R system reduces the false positive rate from $85.5 \%$ to $3.74 \%$, by increasing the detection rate by $5 \%$. For this reason we choose to use this system in this work.

The L\&R system considers four main stages: (i) Generation of local interest points, (ii) Computation of the SIFT descriptors, (iii) SIFT-matching using nearest descriptors, and (iv) Transformation Computation and Hypothesis Rejection Tests. The first three stages are the standard ones proposed by Lowe, while the fourth stage is employed for reducing the number of false matches, giving robustness to the whole system. The procedure for implementing this stage can be seen in [15].

\section{Proposed System for Fingerprint Verification}

The proposed system for fingerprint verification is based on the L\&R wide baseline matching system described in the former section. However, for applying this system in real world harsh conditions (state of the art fingerprint testing protocols) two main improvements are included: (i) a fingerprint enhancement pre-processing module, and (ii) a statistical classification post-processing module.

\subsection{Fingerprint Verification Analysis}

We performed several fingerprint analysis experiments using different fingerprint public databases, and we verified that the L\&R wide baseline system allows matching fingerprints. As an example, in Fig. 1 we show the detected local interest points in a fingerprint's reference-test image pair. In Fig. 2 (a) are shown the corresponding correct matches. As it can be seen, the wide baseline system achieves matching correctly the two fingerprints. In Figs. 1 (b)-(c) are shown some selected matched local interest points. It can be verified that the local interest points do not correspond to minutiae or singular points. As mentioned they correspond to local maxima in the position-scale multi-resolution representation of the fingerprint images. The same is observed in Fig. 1. 
One of main problems in fingerprint verification is the nonlinear distortion in fingerprint images, which disturbs the matching process [14]. This problem is tackled by limiting the acceptable distortion in the acquisition process, by estimating the distortion during the matching process [16][1], or by compensating the distortion using fingerprint warping [14]. The here-proposed wide baseline matching approach is robust against nonlinear distortions in fingerprint images. It can be proved that any differentiable non-linear transformation that is locally near-orthogonal can be approximated by a bundle of local similarity approximations using the Hough transform methodology, if the density of matches between interest points is high enough.
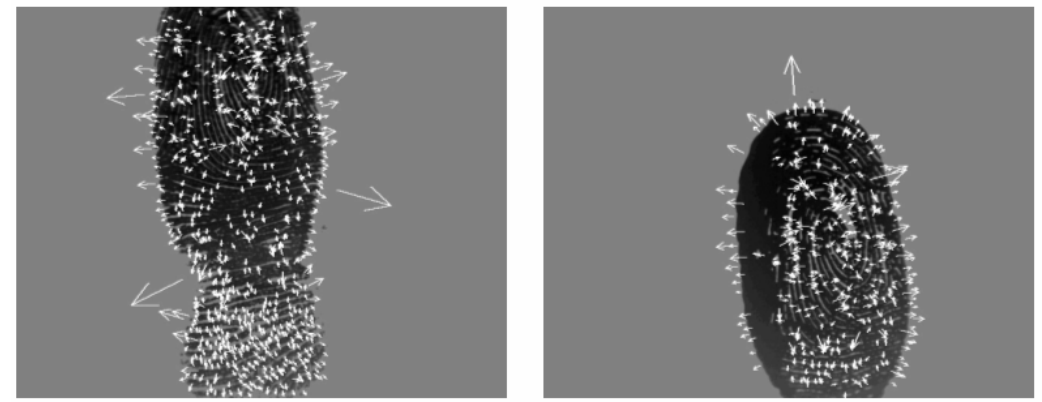

Fig. 1. Example of detected local interest points in a fingerprint's reference-test image pair. Interest points are displayed as arrows, whose origin, orientation and size corresponds to the position $(x, y)$, orientation $\theta$ and scale $s$ of the corresponding interest points.

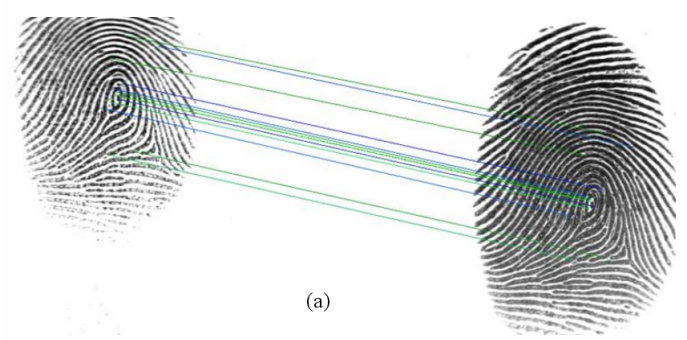

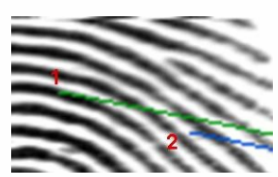

(b)

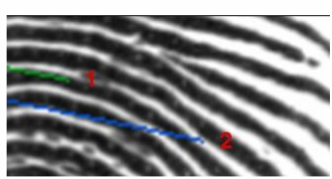

(c)

Fig. 2. (a) Fingerprint's reference-test image pair with matches. (b)-(c) Matched local interest points.

\subsection{Fingerprint Enhancement}

Due to their low quality, fingerprint images are optionally enhanced before applying the wide baseline recognition system. The fingerprint is divided into a 
two-dimensional array of squared regions, and then the local orientation and local frequency are calculated in each region to get a pair of orientation and frequency fields over the complete image. Finally, the pair of fields is used to generate a bank of real-valued Gabor filters, which is applied to the image to enhance it.

\subsection{Bayes Classification}

After applying the L\&R methodology to verification problems in which the fingerprints quality changes largely (changes in finger position, orientation, pressure, skin distortion, etc.), we noted that the number of false positives was still large. We solve this problem by applying a statistical classifier (Naïve Bayes) after the L\&R system. We defined the following 12 features for the classifier (see details in section 2), whose relevance was analyzed using the Weka package [17]:

- TNMatches: Total number of SIFT matches between the reference and test images.

- PTime: Processing time as a measure of the complexity of the matching process.

- NAffinT: Number of detected affine transformations between the reference and test images.

- NMatches: Number of associated SIFT matches included in the best transformation.

- PBT: Probability of the best transformation

- LCorr: Linear correlation parameter of the best transformation

- PCorr: Pixel correlation parameter of the best transformation

- MNDesc: Maximum number of test image descriptors who are matched to the same reference image descriptor, considering the best transformation.

- ScaleAffinMax: Absolute value of the largest eigenvalue of the affine transformation matrix of the best transformation (i.e. largest scale of the best affine transformation).

- ScaleAffinMin: Absolute value of the lowest eigenvalue of the affine transformation matrix of the best transformation (i.e., lowest scale of the best affine transformation).

- NIncMatches: Number of matches of the best transformation who are incompatible with the semi-local constraints.

- RansacPar: RANSAC-compatibility with precise affine transform. A small subset of 3 matches from $B$ is selected to construct a transformation TB who is tested against all the resting matches in $\mathrm{B}$. The matches from $\mathrm{B}$ who are correctly mapped using TB with a very little error are called compatible matches. If more than $2 / 3$ of the matches from $B$ are compatible, the incompatible matches are deleted and a final fine transformation is calculated using the compatible matches. This procedure is tried 10 times using different subsets with 3 matches from B. If in none of the 10 iterations a fine transformation is obtained, RANSAC fails and the initial transformation is preserved.

\section{Preliminary Results}

We present results of the operation of the proposed system for fingerprint verification. Although this system validation is preliminary we choose to use a state of the art 
fingerprint database. We select to use the DB1-DB4 databases from FVC2004. The FVC 2004 test protocol was followed (see details in Cappelli et al. [2]), and Genuine recognition attempts (GNA) and Impostor recognition attempts (IRA) sets for the train and test databases were built. The Naïve Bayes classifier of the proposed fingerprint verification system was trained using the Weka package [17] and 10-fold crossvalidation. The training of the classifier was performed using the DB1-DB4 train datasets. Several tests were executed over the test datasets using different flavors of the proposed system. These flavors were obtained using different enhancement methods, SIFT descriptors of different sizes, and features. Best results were obtained with large descriptors (30x30). The use of large regions in the SIFT descriptor calculation helps to break the local symmetry observed in the fingerprint, and then helps to produce more distinctive descriptors.

The image enhancement pre-processing before the SDoG keypoints calculation, which is a novelty in general-purpose wide-baseline methods, helps to remove acquisition noise, giving a very repeatable fingerprint image, which produces more repeatable keypoints and descriptors, improving the verification results. Slightly different enhancement pre-processing modules were used for each DB, depending on the sensor type used during image acquisition (see table 1). DB1 was binarized using the free software FVS from [18], which uses Gabor filters. DB2 and DB3 where first normalized to zero mean and unitary variance [10], and then binarized. The binarization threshold was set manually, trying to preserve the topography of fingerprint, minutiae and ridges. DB4 was enhanced using the methodology described in section 3.2: normalization and Gabor filtering with parameters automatically tuned using an orientation and a frequency image (similar to [19][21]).

The Bayesian classifier helps to discard a great amount of false detections, which are produced by the fingerprint local symmetry problem mentioned above. This can be illustrated showing that, when using the classifier, the verification results obtained on the $\mathrm{DB} 1$ database are $\mathrm{TP}=79.1 \%$ and $\mathrm{FP}=5.7 \%$ (see table 2), while when not using it are $\mathrm{TP}=98.5 \%$ and $\mathrm{FP}=73.29 \%$. Thus, the main effect of the classifier is to reduce largely the number of false detections.

When comparing the obtained results with the ones from state of the art systems participating in the FVC2004 competition (systems developed by research institutions working for years in fingerprint verification), we observe that our system achieved a competitive performance. We believe that this is very promising because our

Table 1. Selected Features and enhancement method for the classifier training (DB: Databases)

\begin{tabular}{|l|l|l|}
\hline DB & Selected Features & Enhancement Method \\
\hline 1 & PTime, TNMatches, NMatches, PCorr & Gabor implementation from [19]. \\
\hline 2 & PTime, TNMatches, NAffinT, NMatches, PCorr & $\begin{array}{l}\text { Zero mean+unitary variance } \\
\text { normalization+0.2 threshold binarization. }\end{array}$ \\
\hline 3 & PTime, TNMatches, NMatches & $\begin{array}{l}\text { Zero mean+unitary variance } \\
\text { normalization+0.2 threshold binarization. }\end{array}$ \\
\hline 4 & $\begin{array}{l}\text { PTime, TNMatches, PBT, NAffinT, NMatches, PCorr, } \\
\text { LCorr, MNDesc, ScaleAffinMax, ScaleAffinMin, } \\
\text { NIncMatches. }\end{array}$ & Own Gabor Implementation \\
\hline
\end{tabular}


Table 2. Recognition statistics in the FVC2004 databases DB1-DB4

\begin{tabular}{|c|c|c|c|c|c|}
\hline \multirow{2}{*}{ DB } & \multicolumn{3}{|c|}{ Train } & \multicolumn{3}{c|}{ Test } \\
\cline { 2 - 6 } & TP\% (100-FRR) & FP\% (FAR) & TP\% (100-FRR) & FP\% (FAR) & ERR \\
\hline 1 & 86.1 & 15.6 & 79.1 & 5.7 & 13.3 \\
\hline 2 & 68.9 & 13.3 & 73.2 & 14.8 & 20.8 \\
\hline 3 & 86.8 & 3.2 & 72.3 & 3.4 & 15.55 \\
\hline 4 & 84.6 & 4.1 & 78.4 & 17.3 & 19.45 \\
\hline
\end{tabular}

approach is the first one that solves a fingerprint verification problem using a generalpurpose wide-baseline method, and it can still be improved, extended and tuned to achieve state of the art results.

\section{Conclusions}

A new approach to automatic fingerprint verification based in a general-purpose wide baseline matching methodology was proposed. Instead of detecting and matching the standard structural features, in the proposed approach local interest points are detected in the fingerprint, then local descriptors are computed in the neighborhood of these points, and finally these descriptors are matched. Image enhancement as pre-processing module, several verification stages, and a simple statistical classifier are employed for reducing the number of false positives. The nature of the interest points permits to integrate them with minutia points, but a useful way to integrate both information sources need to be investigated. The proposed fingerprint verification system was validated using the FVC2004 test protocol. The system achieves competitive results, without using any a priori knowledge of the finger minutia and singular points information. We expect to improve these results by integrating minutiae-derived descriptors.

\section{References}

1. Bazen, A.M., Gerez, S.H.: Systematic methods for the computation of the directional fields and singular points of fingerprints. IEEE Trans. Pattern Anal. Machine Intell. 24(7), 905919 (2002)

2. Cappelli, R., Maio, D., Maltoni, D., Wayman, J., Jain, A.K.: Performance evaluation of fingerprint verification systems. IEEE Trans. Pattern Anal. Machine Intell. 28(1), 3-18 (2006)

3. Ferrari, V., Tuytelaars, T., Van Gool, L.: Simultaneous Object Recognition and Segmentation by Image Exploration. In: Pajdla, T., Matas, J(G.) (eds.) ECCV 2004. LNCS, vol. 3021, pp. 40-54. Springer, Heidelberg (2004)

4. Harris, C., Stephens, M.: A combined corner and edge detector. In: Proc. 4th Alvey Vision Conf., pp. 147-151. Manchester, UK (1998)

5. Lee, H.C., Gaensslen, R.E.: Advances in Fingerpint Tecnology. Elsevier, NY (1991)

6. Lowe, D.: Local feature view clustering for 3D object recognition. In: IEEE Conference on Computer Vision and Pattern Recognition, Hawaii, pp. 682-688. IEE Press, New York (2001)

7. Lowe, D.: Distinctive Image Features from Scale-Invariant Keypoints. Int. Journal of Computer Vision 60(2), 91-110 (2004) 
8. Loncomilla, P., Ruiz-del-Solar, J.: Gaze Direction Determination of Opponents and Teammates in Robot Soccer. In: Cabonell, J.G., Siekmann, J. (eds.) RoboCup 2005. LNCS (LNAI), vol. 4020, pp. 230-242. Springer, Heidelberg (2006)

9. Loncomilla, P., Ruiz-del-Solar, J.: A Fast Probabilistic Model for Hypothesis Rejection in SIFT-Based Object Recognition. In: Martínez-Trinidad, J.F., Carrasco Ochoa, J.A., Kittler, J. (eds.) CIARP 2006. LNCS, vol. 4225, pp. 696-705. Springer, Heidelberg (2006)

10. Maltoni, D., Maio, D., Jain, A.K., Prabhakar, S.: Handbook of Fingerprint Recognition. Springer, NY (2003)

11. Mikolajczyk, K., Schmid, C.: Scale \& Affine Invariant Interest Point Detectors. Int. Journal of Computer Vision 60(1), 63-96 (2004)

12. Mikolajczyk, K., Schmid, C.: A performance evaluation of local descriptors. IEEE Trans. Pattern Anal. Machine Intell. 27(10), 1615-1630 (2005)

13. Mikolajczyk, K., Tuytelaars, T., Schmid, C., Zisserman, A., Matas, J., Schaffalitzky, F., Kadir, T., Van Gool, L.: A Comparison of Affine Region Detectors. Int. Journal of Computer Vision 65(1-2), 43-72 (2005)

14. Ross, A., Dass, S., Jain, A.K.: Fingerprint warping using ridge curve correspondences. IEEE Trans. Pattern Anal. Machine Intell. 28(1), 19-30 (2006)

15. Ruiz-del-Solar, J., Devia, C., Loncomilla, P., Concha, F.: Offline Signature Verification using Local Interest Points and Descriptors. In: Proc. CIARP 2008. LNCS, vol. 5197. Springer, Heidelberg (2008)

16. Schaffalitzky, F., Zisserman, A.: Automated location matching in movies. Computer Vision and Image Understanding 92(2-3), 236-264 (2003)

17. Kovacs-Vajna, Z.M.: A fingerprint verification system based on triangular matching and dynamic time warping. IEEE Trans. Pattern Anal. Machine Intell. 22(11), 1266-1276 (2000)

18. Weka website, http://www.cs.waikato.ac.nz/ml/weka/

19. FVS (2003), http: / / fvs. sourceforge. net

20. Hong, L., Wan, Y., Jain, A.: Fingerprint image enhancement: Algorithm and performance evaluation. IEEE Transactions on Pattern Analysis and Machine Intelligence 20(8), 777 789 (1998)

21. Wang, Y., Hu, J., Han, F.: Enhanced gradient-based algorithm for the estimation of fingerprint orientation fields. Applied Mathematics and Computation 185, 823-833 (2007)

22. Prabhakar, S., Jain, A.K., Pankanti, S.: Learning fingerprint minutiae location and type. Pattern Recognition 36, 1847-1857 (2003) 